\title{
BIOLOGICAL ASPECTS OF LEPIDOGLYPHUS DESTRUCTOR (SCHRANK) (ACARI: ASTIGMATA: GLYCYPHAGIDAE) REARED UNDER THREE TEMPERATURE DEGREES AND DIETS
}

\author{
E. M. A. Yassin, Asmaa, R. Abd El-Khali, M.M. El-Sebaay \\ and Safinaz A. Abd El-Aziz \\ Plant Protection Research Institute, Agricultural Research Center, Dokki, Giza, Egypt
}

Received: Mar. 30,2017

Accepted: Apr. 18, 2017

\begin{abstract}
The glycyphagid mite, Lepidoglyphus destructor (Schrank) individuals were extracted from wheat and broad bean hay at El-Menofia Governorate and reared on different stored hay at three temperature degrees. Biological experiments and observations were conducted in the laboratory under controlled conditions of $20{ }^{\circ} \mathrm{C}, 25{ }^{\circ} \mathrm{C}$ and $30{ }^{\circ} \mathrm{C}$ and relative humidity $80 \%$ R.H. Broad bean and wheat hay were used singly and mixed with dry yeast as a mite food. The obtained results showed that developmental stages, longevity and life span and fecundity were obviously affected at different diets and temperature degrees. The highest life span period was recorded with female fed on mixture of broad bean and yeast averaged as 49.4 days at $20 \stackrel{\circ}{\circ}$, but the shortest period was recorded for male individuals at $30{ }^{\circ} \mathrm{C}$ when reared on the mixture of broad bean and wheat hay as 20.0 day. Mixture of broad bean hay \& yeast proved to be the suitable diets resulting the highest number of deposited eggs at $30{ }^{\circ} \mathrm{C}$ recorded 159.9 eggs but the lowest number was noticed for $L$. destructor females at $20{ }^{\circ} \mathrm{C}$ when the individuals fed on mixture of broadbean and wheat hay (82.2 eggs) at $20{ }^{\circ} \mathrm{C}$. The current study provided evidence of the nutritive attractiveness of broad bean hay mixed with dry yeast for $L$. destructor as a preferable feeding source for laboratory rearing of this astigmatid mite. The obtained results denoted that males took the shortest biological stages (incubation period, life cycle, longevity and life span) than female individuals at tested temperature degrees, where 20 ${ }^{\circ} \mathrm{C}$ was the suitable one and increased these periods.
\end{abstract}

Key words: longevity, life span, life cycle, hay, mites, glycyphagid mite.

\section{INTRODUCTION}

Up-to-date dramatically increase in the population in the world requires an efficient modern human food and animal production industry and the manufacture of good quality feeds and food. Damage by insects, mites, fungi, and sprouting causes hundreds of millions of dollars of economic losses to grain producers, merchandisers, and processors each year (Harein and Meronuck1995).Stored product mites are important pests of stored food commodities and animal feed in areas with humid climates (Sanchez-Ramos and Castanera 2003). These pests negatively influence the quality of stored commodities, cause allergic reaction (Kondreddi et al., 2006) and disseminate toxic moulds (Hubert et al., 2004). Majority of members of the glycyphagid mites (Glycyphagidae) are cosmopolitan, commonly known as synanthropic mite species. Of them, the mite, Lepidoglyphus destructor (Schrank) is one of the commonest species of stored product mites and is frequently found in association with other mites i.e. Acarus siro and Cheyletus eruditus or C. malaccensis (Hughes, 1976), in soil samples (Sheals, 1956), stacks of grain, straw, and hay standing in the open field or in a permanent stackyard (Griffith, 1960), linseed, rice, dried fruits, sugar beet seed (Chmielewski, 1969), dried calves stomachs, dead insects, dried mammal skins, rodent and bumble bee nests (Hughes (1976) and Post-harvest sweepings (hay, straw) from barn, Chmielewski (2001). Some studies presented results of biological investigations on $L$. destructor fed various kinds of nutrients (bee-bread, pollen, wheat germ, 
yeast) (Chmielewski, 1977, 1987; Cornete and Kahl, 1996; Knulle, 1991; Matsumoto, 1963; Parkinson, 1989; Stratil et al., 1980) but there are no data on its feeding and development on stored product hay. The present work aims to study the effect of different types of food on biological aspects, life cycle, longevity, life span and fecundity of astigmatid mite, Lepidoglyphus destructor at different laboratory conditions.

\section{MATERIALS AND METHODS}

The glycyphagid mite, $L$. destructor (Schrank) individuals were collected from wheat hay at El-Menofia Governorate (Ashmoun district). Samples of wheat hay (500 gm) were randomized obtained from open fields and different animal's stockades and put in polyethylene bags, then transferred to laboratory for extraction and identification.

Pure culture. For preparing pure culture of tested mites, plastic cups of $(1.5 \mathrm{~cm}$ high $x 2.5 \mathrm{~cm}$ in diameter) were filled up to 0.5 $\mathrm{cm}$ with plaster of Paris and activated charcoal in the rate of 8: 2 , respectively. One adult female and male of $L$. destructor were placed in the prepared cup, supplied with food and drops of water added to maintain suitable relative humidity and kept in an incubator at $25^{\circ} \mathrm{C}$. For individual rearing, ten newly deposited eggs were transferred from the mother culture singly one to every rearing plastic cell $(1.5 \mathrm{~cm}$ high $\times 2.5 \mathrm{~cm}$ in diameter). Each newly hatched larva was supplied with food kept till reaching maturity. Mites were examined twice daily. Observations concerning all biological aspects were recorded allover the life of mites at 20,25 and $30{ }^{\circ} \mathrm{C}$ and $80 \%$ R.H.

Source of food: The following diets were used in the experiments: Broad bean hay only, broad bean hay mixed with dry yeast, wheat hay only, wheat hay mixed with dry yeast and broad bean hay mixed with wheat hay.

Statistical analysis: All obtained data were subjected to one-way analysis of variance (ANOVA) and means were separated by Duncan's multiple range test (Duncan ,1955).

\section{RESULTS AND DISCUSSION Habitat and behavior:}

The glycyphagid mite, Lepidoglyphus destructor was found in large numbers association with stored wheat and broadbean hay at Ashmoun district (ElMenofia Governorate). This mite passed through four different stages before reaching adult (egg, larva, protonymphs, and tritonymph).When full-grown, every moving immature stage enter to quiescent stage in which it seeks a dry or crack in the substrate of rearing chamber and completely ceases feeding and movement. Newly emerged individual kept quite beside its old skin for a short time, then started to move actively searching its food after 8-12 minutes. Young males and females started to copulate soon after eclosion. The time of copulation was 15-20 minutes and copulation was repeated many times. The adult female of $L$. destructor began to lay eggs 5- 7 days after copulation and finished the oviposition process about 7- 8 days before their natural death.

\section{A- Lepidoglyphus destructor female Incubation period:}

The newly deposited eggs of $L$. destructor in cracks and rearing substrate were elongated and translucent when deposited, then changes to dark whitish color before hatching. During hatching,, the egg rupture longitudinally and larva crawled out from the medium slit with its hind legs at first, then larva move outside to search for food. The hatching process lasted about 1820 minutes. The shortest incubation period of the mite $L$. destructor was recorded when the mite fed on broadbean hay mixed with wheat hay (3.0 days) at $30{ }^{\circ} \mathrm{C}$, while the longest period was noticed when the mite individuals fed on broadbean hay mixed with yeast (5.5 days) at $20{ }^{\circ} \mathrm{C}$, with L.S. D. at $0.05=0.06$ for diets and 0.04 for temperature effect, Table (1). 
Biological aspects of lepidoglyphus destructor (schrank) (acari: astigmata:....

Table (1): Mean duration of Lepidoglyphus destructor female when fed on different diets at temperature degrees

\begin{tabular}{|c|c|c|c|c|c|c|c|}
\hline \multirow{2}{*}{$\begin{array}{c}\text { Biological } \\
\text { aspect }\end{array}$} & \multicolumn{5}{|c|}{ Diets } & \multicolumn{2}{|c|}{$\begin{array}{l}\text { L.S.D. at } \\
0.05 \text { level }\end{array}$} \\
\hline & $\begin{array}{l}\text { B.b. h. + } \\
\text { yeast }\end{array}$ & B. b. $h$ & $\begin{array}{l}\text { W.h. + } \\
\text { yeast }\end{array}$ & W. h. & B.b $h+w \cdot h$ & Diets & Tem \\
\hline \multicolumn{8}{|c|}{$20^{\circ} \mathrm{C}$} \\
\hline $\begin{array}{l}\text { Incubation } \\
\text { period }\end{array}$ & $\begin{array}{c}5.5 \pm 0.2 \\
(5.2-5.8)\end{array}$ & $\begin{array}{c}5.0 \pm 0.2 \\
(4.7-5.3)\end{array}$ & $\begin{array}{c}4.4 \pm 0.2 \\
(4.2-4.6)\end{array}$ & $\begin{array}{l}3.98 \pm 0.1 \\
(3.8-4.2)\end{array}$ & $\begin{array}{l}3.58 \pm 0.1 \\
(3.5-3.7)\end{array}$ & 0.06 & 0.04 \\
\hline Life cycle & $\begin{array}{c}16.1 \pm 0.3 \\
(15.5-16.6)\end{array}$ & $\begin{array}{c}15.5 \pm 0.1 \\
(15.3-15.6)\end{array}$ & $\begin{array}{c}14.7 \pm 0.2 \\
(144-14.9)\end{array}$ & $\begin{array}{c}13.9 \pm 0.1 \\
(13.7-14.1)\end{array}$ & $\begin{array}{c}13.0 \pm 0.1 \\
(12.7-13.2)\end{array}$ & 0.16 & 0.12 \\
\hline Longevity & $\begin{array}{c}33.3 \pm 0.7 \\
(32-34)\end{array}$ & $\begin{array}{c}31.1 \pm 0.6 \\
(30-32)\end{array}$ & $\begin{array}{c}28.0 \pm 0.1 \\
(27.9-28.3)\end{array}$ & $\begin{array}{l}27.0 \pm 0.3 \\
(26.4-27)\end{array}$ & $\begin{array}{c}25.1 \pm 0.5 \\
(23.9-25.8)\end{array}$ & 0.35 & 0.27 \\
\hline Life span & $\begin{array}{l}49.4 \pm 0.5 \\
(48.5-50)\end{array}$ & $\begin{array}{c}46.6 \pm 0.6 \\
(45.3-47.5)\end{array}$ & $\begin{array}{c}42.7 \pm 0.2 \\
(42.4-42.9)\end{array}$ & $\begin{array}{c}40.5 \pm 0.4 \\
(39.7-41.1)\end{array}$ & $\begin{array}{c}38.0 \pm 0.5 \\
(36.9-38.6)\end{array}$ & 0.37 & 0.29 \\
\hline \multicolumn{8}{|c|}{$25 \stackrel{\circ}{\circ}$} \\
\hline $\begin{array}{l}\text { Incubation } \\
\text { period }\end{array}$ & $\begin{array}{c}5.15 \pm 0.1 \\
(5-5.3)\end{array}$ & $\begin{array}{l}4.52 \pm 0.1 \\
(4.4-4.8)\end{array}$ & $\begin{array}{c}4.01 \pm 0.1 \\
(3.9-.2)\end{array}$ & $\begin{array}{c}3.79 \pm 0.8 \\
(3.6-3.9)\end{array}$ & $\begin{array}{c}3.4 \pm 0.1 \\
(3.2-3.6)\end{array}$ & 0.06 & 0.04 \\
\hline Life cycle & $\begin{array}{c}15.1 \pm 0.4 \\
(14.5-16)\end{array}$ & $\begin{array}{c}14.7 \pm 0.2 \\
(14.3-15)\end{array}$ & $\begin{array}{l}13.8 \pm 0.1 \\
(13.6-14)\end{array}$ & $\begin{array}{c}12.6 \pm 0.1 \\
(12.5-12.9)\end{array}$ & $\begin{array}{c}11.6 \pm 0.1 \\
(11.5-11.7)\end{array}$ & 0.16 & 0.12 \\
\hline Longevity & $\begin{array}{l}30.9 \pm 0.8 \\
(28.9-32)\end{array}$ & $\begin{array}{c}28.1 \pm 0.2 \\
(27.9-28.6)\end{array}$ & $\begin{array}{c}24.4 \pm 0.7 \\
(24-26)\end{array}$ & $\begin{array}{c}25.0 \pm 0.8 \\
(24-26)\end{array}$ & $\begin{array}{c}21.9 \pm 0.9 \\
(20-23)\end{array}$ & 0.35 & 0.27 \\
\hline Life span & $\begin{array}{l}45.9 \pm 0.9 \\
(43.9-47)\end{array}$ & $\begin{array}{c}42.8 \pm 0.3 \\
(42.5-43.5)\end{array}$ & $\begin{array}{c}38.22 \pm 0.7 \\
(37.8-39.9)\end{array}$ & $\begin{array}{c}37.6 \pm 0.8 \\
(36.6-38.6)\end{array}$ & $\begin{array}{c}33.4 \pm 1.0 \\
(31.5-34.6)\end{array}$ & 0.37 & 0.29 \\
\hline \multicolumn{8}{|c|}{$30 \stackrel{\circ}{\circ}$} \\
\hline $\begin{array}{l}\text { Incubation } \\
\text { period }\end{array}$ & $\begin{array}{c}4.8 \pm 0.1 \\
(4.7-4.9)\end{array}$ & $\begin{array}{c}4.4 \pm 0.1 \\
(4.2-4.6)\end{array}$ & $\begin{array}{c}3.8 \pm 0.1 \\
(3.6-3.9)\end{array}$ & $\begin{array}{c}3.5 \pm 0.1 \\
(3.4-3.6)\end{array}$ & $\begin{array}{c}3.0 \pm 0.1 \\
(2.9-3.2)\end{array}$ & 0.06 & 0.04 \\
\hline Life cycle & $\begin{array}{c}13.5 \pm 1.0 \\
(12-15)\end{array}$ & $\begin{array}{c}13.8 \pm 0.1 \\
(13.4-13.9)\end{array}$ & $\begin{array}{c}13.0 \pm 0.1 \\
(12.8-13.1)\end{array}$ & $\begin{array}{c}12.0 \pm 0.1 \\
(11.9-12.2)\end{array}$ & $\begin{array}{c}11.0 \pm 0.1 \\
(10.8-11.2)\end{array}$ & 0.16 & 0.12 \\
\hline Longevity & $\begin{array}{c}28.0 \pm 0.9 \\
(26-29)\end{array}$ & $\begin{array}{c}24.9 \pm 0.7 \\
(24-26)\end{array}$ & $\begin{array}{c}21.2 \pm 1.0 \\
(20-23)\end{array}$ & $\begin{array}{c}21.9 \pm 0.7 \\
(21-23)\end{array}$ & $\begin{array}{c}19.3 \pm 0.4 \\
(18.9-20)\end{array}$ & 0.35 & 0.27 \\
\hline Life span & $\begin{array}{c}41.5 \pm 1.3 \\
(39-43)\end{array}$ & $\begin{array}{c}38.7 \pm 0.8 \\
(37.7-39.9)\end{array}$ & $\begin{array}{l}33.9 \pm 0.8 \\
(33-35.8)\end{array}$ & $\begin{array}{l}33.7 \pm 0.8 \\
(33-35.2)\end{array}$ & $\begin{array}{l}30.3 \pm 0.4 \\
(29.8-31)\end{array}$ & 0.37 & 0.29 \\
\hline
\end{tabular}

B. b. $h=$ broad bean hay $W . h .=$ wheat hay 


\section{Life cycle:}

Observations of feeding and life cycles of L. destructor female on different introduced diets gave evidence that these stored materials were acceptable by this mite for nourishment, Table (1). The longest duration of $L$. destructor averaged 16.1 days when the mite females fed on mixture of broadbean hay with dry yeast at $20 \stackrel{\circ}{ } \mathrm{C}$, as, it significantly decreased when mixture broadbean hay with wheat hay was introduced as feeding source for the mite recording 11.0 days at $30{ }^{\circ} \mathrm{C}$. The statistical analysis of obtained data showed that L.S. D. at 0.05 level was 0.16 and 0.12 for effect of different diets and temperatures on the life cycle of $L$. destructor.

\section{Longevity:}

The tested temperatures and food sources were obviously affected the longevity of the adult female of $L$. destructor, Table (1). Adult female longevity decreased when temperature increased from 20 to $30 \stackrel{\circ}{\circ}$. It lived for 33.3 days (the longest period) at 20 ${ }^{\circ} \mathrm{C}$ when the mites reared on mixture of broad bean hay and yeast and decreased to recorded 19.3 days (the shortest period) when the mites fed on the mixture of broadbean and wheat hay at $30{ }^{\circ} \mathrm{C}$. The statistical analysis of obtained data indicated that L.S. D. at 0.05 level $=0.35$ and 0.27 for effect of diets and temperature on the mite female longevity, respectively.

\section{a- Preoviposition period:}

Statistical analysis of data tabulated in Table (2) proved that this period was affected by the type of food. Accordingly, this period was 6.0, 5.6, 5.1, 4.6 and 4.2 days (longest periods) when the female fed at $20{ }^{\circ} \mathrm{C}$ on broadbean mixed with yeast, broadbean hay, wheat hay mixed with yeast, wheat hay and broadbean hay mixed with wheat hay, respectively, and these periods decreased at $30{ }^{\circ} \mathrm{C}$ to recorded 4.8, 5.0, 4.1, 4.0 and 3.6 days (the shortest periods) when
L. destructor female reared on the same trend of previously mentioned diets, respectively.

\section{b- Oviposition period:}

The introduced food suitability clearly affects the oviposition period of $L$. destructor, Table (2). However, feeding of mite female at $20{ }^{\circ} \mathrm{C}$ on mixture of broadbean hay and yeast obviously prolonged the oviposition period (19.7 days) than any diets and temperatures. This period took the shortest period (11 days) when the female fed on mixture of broadbean hay and wheat hay at $30{ }^{\circ} \mathrm{C}$. The significant differences occurred between all feeding diets at different temperature (L.S.D. at 0.05 $=0.39$ and 0.30 , respectively).

\section{c- Postoviposition period:}

Data concerning the response of the postoviposition period of $L$. destructor female to different feeding diets are tabulated in Table (2). The mean period of postoviposition was high when the female mites fed on broadbean hay mixed with yeast, recorded 7.7 days at $20{ }^{\circ} \mathrm{C}$. changed to 4.7 days (the shortest period) when mites reared on the mixture of broadbean and wheat hay. L.S.D. at 0.05 level $=0.11$ and 0.09 for diets and temperature effect, respectively.

\section{Life span:}

Accordingly, the life span of the astigmatid mite, $L$. destructor female also affected by feeding on different introduced diets as shown in Table (1). The female life span of mites resulted from feeding on mixture of broadbean hay with yeast was high at $20 \stackrel{\circ}{\circ}$, recorded 49.4 days which remarkably decreased to 30.3 days when the mites fed on mixture of broad bean and wheat hay at $30 \stackrel{\circ}{\circ}$. (L.S.D. at 0.05 level $=$ 0.37 and 0.29 in case of diets and temperature effects, respectively). 
Biological aspects of lepidoglyphus destructor (schrank) (acari: astigmata:....

Table (2): Longevity and fecundity (number of eggs) of Lepidoglyphus destructor female when fed on different diets and temperature degrees

\begin{tabular}{|c|c|c|c|c|c|c|c|}
\hline \multirow{2}{*}{$\begin{array}{l}\text { Biological } \\
\text { aspect }\end{array}$} & \multicolumn{5}{|c|}{ Diets } & \multicolumn{2}{|c|}{$\begin{array}{l}\text { L.S.D. at } \\
0.05 \text { level }\end{array}$} \\
\hline & $\begin{array}{c}\text { B.b. h. + } \\
\text { yeast }\end{array}$ & B. b. h & $\begin{array}{l}\text { W.h. + } \\
\text { yeast }\end{array}$ & W. h. & B.b $h+w \cdot h$ & Diets & Tem. \\
\hline \multicolumn{8}{|c|}{$20 \stackrel{\circ}{C}$} \\
\hline $\begin{array}{l}\text { Preoviposit- } \\
\text { ion period }\end{array}$ & $\begin{array}{c}6.0 \pm 0.1 \\
(5.8-6.2)\end{array}$ & $\begin{array}{c}5.6 \pm 0.2 \\
(5-5.9) \\
\end{array}$ & $\begin{array}{c}5.1 \pm 0.2 \\
(5-5.5)\end{array}$ & $\begin{array}{c}4.6 \pm 0.2 \\
(4.3-4.9)\end{array}$ & $\begin{array}{c}4.2 \pm 0.1 \\
(4-4.2)\end{array}$ & 0.07 & 0.05 \\
\hline $\begin{array}{l}\text { Oviposition } \\
\text { period }\end{array}$ & $\begin{array}{c}19.7 \pm 0.8 \\
(18.5-21.1)\end{array}$ & $\begin{array}{c}18.5 \pm 0.8 \\
(17.4-19.6)\end{array}$ & $\begin{array}{c}16.6 \pm 0.4 \\
(16.1-17.3)\end{array}$ & $\begin{array}{c}16.2 \pm 0.5 \\
(15.6-17.1)\end{array}$ & $\begin{array}{c}15.5 \pm 0.6 \\
(14.2-16.1)\end{array}$ & 0.39 & 0.30 \\
\hline $\begin{array}{l}\text { Postovipos- } \\
\text { ition period }\end{array}$ & $\begin{array}{c}7.7 \pm 0.4 \\
(7-8.2) \\
\end{array}$ & $\begin{array}{c}7.1 \pm 0.2 \\
(6.8-7.5) \\
\end{array}$ & $\begin{array}{c}6.4 \pm 0.2 \\
(6-6.5) \\
\end{array}$ & $\begin{array}{c}5.9 \pm 0.2 \\
(5.4-6) \\
\end{array}$ & $\begin{array}{c}5.5 \pm 0.1 \\
(5.3-5.6) \\
\end{array}$ & 0.11 & 0.09 \\
\hline Fecundity & $\begin{array}{l}133.3 \pm 4.4 \\
(127-140)\end{array}$ & $\begin{array}{l}124.8 \pm 1.3 \\
(122-127)\end{array}$ & $\begin{array}{c}109.9 \pm 1.6 \\
(107-112)\end{array}$ & $\begin{array}{c}92.4 \pm 3.5 \\
(89-99)\end{array}$ & $\begin{array}{c}82.2 \pm 1.8 \\
(80-85)\end{array}$ & 1.06 & 0.82 \\
\hline \multicolumn{8}{|c|}{$25 \stackrel{\circ}{C}$} \\
\hline $\begin{array}{l}\text { Preoviposit- } \\
\text { ion period }\end{array}$ & $\begin{array}{c}5.0 \pm 0.1 \\
(4.8-5.3) \\
\end{array}$ & $\begin{array}{l}5.2 \pm 0.1 \\
(5-5.4) \\
\end{array}$ & $\begin{array}{c}4.6 \pm 0.1 \\
(4.4-4.7) \\
\end{array}$ & $\begin{array}{c}4.3 \pm 0.1 \\
(4.2-4.6) \\
\end{array}$ & $\begin{array}{c}4.0 \pm 0.1 \\
(3.8-4.2) \\
\end{array}$ & 0.07 & 0.05 \\
\hline $\begin{array}{l}\text { Oviposition } \\
\text { period }\end{array}$ & $\begin{array}{c}19.1 \pm 1.0 \\
(16.9-20.2) \\
\end{array}$ & $\begin{array}{c}16.0 \pm 0.3 \\
(15.8-16.5) \\
\end{array}$ & $\begin{array}{c}13.7 \pm 0.6 \\
(12.9-15.2) \\
\end{array}$ & $\begin{array}{c}15.2 \pm 0.9 \\
(13.9-16.3) \\
\end{array}$ & $\begin{array}{r}12.7 \pm 1.1 \\
(11-14.1) \\
\end{array}$ & 0.39 & 0.30 \\
\hline $\begin{array}{l}\text { Postovipos- } \\
\text { ition period }\end{array}$ & $\begin{array}{c}6.8 \pm 0.5 \\
(6-7.6) \\
\end{array}$ & $\begin{array}{c}6.9 \pm 0.1 \\
(6.6-7.1)\end{array}$ & $\begin{array}{c}6.2 \pm 0.3 \\
(6-6.7) \\
\end{array}$ & $\begin{array}{r}5.5 \pm 0.1 \\
(5.4-5.6) \\
\end{array}$ & $\begin{array}{c}5.0 \pm 0.1 \\
(4.7-5.3)\end{array}$ & 0.11 & 0.09 \\
\hline Fecundity & $\begin{array}{l}149.3 \pm 1.9 \\
(146-152)\end{array}$ & $\begin{array}{r}134.6 \pm 2.0 \\
(130-137)\end{array}$ & $\begin{array}{c}124.9 \pm 1.4 \\
(122-127)\end{array}$ & $\begin{array}{l}105.4 \pm 2.1 \\
(103-109)\end{array}$ & $\begin{array}{c}92.2 \pm 1.5 \\
(90-95)\end{array}$ & 1.06 & 0.82 \\
\hline \multicolumn{8}{|c|}{$30 \stackrel{\circ}{ } \mathrm{C}$} \\
\hline $\begin{array}{l}\text { Preoviposit- } \\
\text { ion period }\end{array}$ & $\begin{array}{c}4.8 \pm 0.1 \\
(4.6-4.9) \\
\end{array}$ & $\begin{array}{c}5.0 \pm 0.1 \\
(4.8-5.2) \\
\end{array}$ & $\begin{array}{c}4.1 \pm 0.1 \\
(4-4.3) \\
\end{array}$ & $\begin{array}{c}4.0 \pm 0.1 \\
(3.8-4.1) \\
\end{array}$ & $\begin{array}{l}3.6 \pm 0.1 \\
(3.5-3.7) \\
\end{array}$ & 0.07 & 0.05 \\
\hline $\begin{array}{l}\text { Oviposition } \\
\text { period }\end{array}$ & $\begin{array}{c}16.7 \pm 1.1 \\
(14.4-17.9)\end{array}$ & $\begin{array}{c}13.4 \pm 0.8 \\
(12.3-14.5)\end{array}$ & $\begin{array}{c}12.1 \pm 1.1 \\
(10.6-14.1)\end{array}$ & $\begin{array}{l}12.9 \pm 0.8 \\
(12-14.1)\end{array}$ & $\begin{array}{c}11.0 \pm 0.4 \\
(10.5-11.7)\end{array}$ & 0.39 & 0.30 \\
\hline $\begin{array}{l}\text { Postovipos- } \\
\text { ition period }\end{array}$ & $\begin{array}{c}6.5 \pm 0.1 \\
(6.3-6.8) \\
\end{array}$ & $\begin{array}{c}6.5 \pm 0.1 \\
(6.4-6.6) \\
\end{array}$ & $\begin{array}{l}5.0 \pm 0.1 \\
(4.8-5.2) \\
\end{array}$ & $\begin{array}{c}5.0 \pm 0.1 \\
(4.8-5.3) \\
\end{array}$ & $\begin{array}{c}4.7 \pm 0.1 \\
(4.6-4.8) \\
\end{array}$ & 0.11 & 0.09 \\
\hline Fecundity & $\begin{array}{l}159.9 \pm 1.3 \\
(157-162)\end{array}$ & $\begin{array}{r}144.7 \pm 1.2 \\
(142-146)\end{array}$ & $\begin{array}{l}130.4 \pm 2.1 \\
(127-134)\end{array}$ & $\begin{array}{l}110.0 \pm 1.2 \\
(109-113)\end{array}$ & $\begin{array}{l}99.9 \pm 1.0 \\
(98-101)\end{array}$ & 1.06 & 0.82 \\
\hline
\end{tabular}

B. b. $\mathrm{h}=$ broad bean hay $\mathrm{W} . \mathrm{h} .=$ wheat hay

\section{B- Lepidoglyphus destructor male Incubation period:}

The respective durations of incubation period of $L$. destructor male when reared on five types of food are summarized in Table (3). Highly food type and temperature effects were observed on this period. Male mites reared on mixture of broadbean hay and yeast had the longest incubation period, which was significantly different from those on the rest of rearing diets, where it 4.0 days at $20{ }^{\circ} \mathrm{C}$, and remarkably decreased when fed on mixture of broadbean and wheat hay at $30{ }^{\circ} \mathrm{C}$, and lasted 2.5 day. The statistical analysis of obtained data indicted that L.S.D. at 0.05 level for effect of different diets and 
Yassin, et al.,

temperature was 0.06 and 0.04 , destructor male developed faster on mixture respectively. of broadbean and wheat hay at $30{ }^{\circ} \mathrm{C}$, and

Life cycle:

The egg-to-adult developmental duration of $L$. destructor male individuals was significantly different among the feeding sources. According to the present study, $L$. recorded 7.8 days, while it increased to the longest level when fed on mixture of broadbean hay with yeast at $20{ }^{\circ} \mathrm{C}$, and durated 13.0 days.

Table (3): Mean duration of Lepidoglyphus destructor male when fed on different diets at different temperature

\begin{tabular}{|c|c|c|c|c|c|c|c|}
\hline \multirow{2}{*}{$\begin{array}{c}\text { Biological } \\
\text { aspect }\end{array}$} & \multicolumn{5}{|c|}{ Diets } & \multicolumn{2}{|c|}{$\begin{array}{l}\text { L.S.D. at } \\
0.05 \text { level }\end{array}$} \\
\hline & $\begin{array}{c}\text { B.b. h. + } \\
\text { yeast }\end{array}$ & B. b. $h$ & $\begin{array}{l}\text { W.h. + } \\
\text { yeast }\end{array}$ & W. h. & B.b $h+w \cdot h$ & Diets & Tem \\
\hline \multicolumn{8}{|c|}{$20^{\circ} \mathrm{C}$} \\
\hline $\begin{array}{l}\text { Incubation } \\
\text { period }\end{array}$ & $\begin{array}{c}4.0 \pm 0.1 \\
(3.8-4.2)\end{array}$ & $\begin{array}{c}3.8 \pm 0.1 \\
(3.6-3.9)\end{array}$ & $\begin{array}{l}3.4 \pm 0.1 \\
(3.3-3.5)\end{array}$ & $\begin{array}{c}3.1 \pm 0.1 \\
(3-3.2)\end{array}$ & $\begin{array}{c}2.9 \pm 0.2 \\
(2.4-3.1)\end{array}$ & 0.06 & 0.04 \\
\hline Life cycle & $\begin{array}{c}13.0 \pm 0.3 \\
(12.5-13.5)\end{array}$ & $\begin{array}{l}12.3 \pm 0.2 \\
(12-12.6)\end{array}$ & $\begin{array}{l}11.4 \pm 0.2 \\
(11-11.5)\end{array}$ & $\begin{array}{c}10.4 \pm 0.2 \\
(10-10.6)\end{array}$ & $\begin{array}{c}9.4 \pm 0.2 \\
(9-9.6)\end{array}$ & 0.11 & 0.08 \\
\hline Longevity & $\begin{array}{c}22.0 \pm 1.1 \\
(20-24)\end{array}$ & $\begin{array}{c}20.0 \pm 1.0 \\
(18-21)\end{array}$ & $\begin{array}{c}17.4 \pm 0.7 \\
(16-18)\end{array}$ & $\begin{array}{c}16.0 \pm 0.2 \\
(15.7-16.3)\end{array}$ & $\begin{array}{c}14.2 \pm 0.3 \\
(13.8-14.6)\end{array}$ & 0.3 & 0.2 \\
\hline Life span & $\begin{array}{c}35.0 \pm 1.2 \\
(32.5-37.0)\end{array}$ & $\begin{array}{c}32.1 \pm 0.9 \\
(30.4-33.6)\end{array}$ & $\begin{array}{c}28.6 \pm 0.8 \\
(27.4-29.5)\end{array}$ & $\begin{array}{c}26.3 \pm 0.3 \\
(25.8-26.6)\end{array}$ & $\begin{array}{l}23.5 \pm 0.4 \\
(23-24.1)\end{array}$ & 1.18 & 0.9 \\
\hline \multicolumn{8}{|c|}{$25 \stackrel{\circ}{\circ}$} \\
\hline $\begin{array}{l}\text { Incubation } \\
\text { period }\end{array}$ & $\begin{array}{l}3.6 \pm 0.04 \\
(3.5-3.6)\end{array}$ & $\begin{array}{c}3.2 \pm 0.1 \\
(3-3.4)\end{array}$ & $\begin{array}{r}3.1 \pm 0.1 \\
(3-3.2)\end{array}$ & $\begin{array}{r}2.8 \pm 0.1 \\
(2.6-3)\end{array}$ & $\begin{array}{c}2.8 \pm 0.1 \\
(2.6-3)\end{array}$ & 0.06 & 0.04 \\
\hline Life cycle & $\begin{array}{r}12.4 \pm 0.2 \\
(12-12.6)\end{array}$ & $\begin{array}{l}11.9 \pm 0.1 \\
(11.8-12)\end{array}$ & $\begin{array}{c}10.5 \pm 0.1 \\
(10.3-10.7)\end{array}$ & $\begin{array}{c}9.5 \pm 0.1 \\
(9.3-9.7)\end{array}$ & $\begin{array}{c}8.5 \pm 0.1 \\
(8.3-8.7)\end{array}$ & 0.11 & 0.08 \\
\hline Longevity & $\begin{array}{c}18.5 \pm 0.7 \\
(17-19)\end{array}$ & $\begin{array}{c}18.0 \pm 0.7 \\
(17-19)\end{array}$ & $\begin{array}{c}15.0 \pm 0.7 \\
(14-16)\end{array}$ & $\begin{array}{l}14.8 \pm 0.1 \\
(14.6-15)\end{array}$ & $\begin{array}{c}13.5 \pm 0.3 \\
(13-14)\end{array}$ & 0.3 & 0.2 \\
\hline Life span & $\begin{array}{c}31.0 \pm 0.8 \\
(29.4-31.6)\end{array}$ & $\begin{array}{l}29.9 \pm 0.7 \\
(28.8-31)\end{array}$ & $\begin{array}{c}25.4 \pm 0.7 \\
(24.5-26.5)\end{array}$ & $\begin{array}{c}24.3 \pm 0.1 \\
(24.1-24.4)\end{array}$ & $\begin{array}{c}22.0 \pm 0.3 \\
(21.4-22.5)\end{array}$ & 1.18 & 0.9 \\
\hline \multicolumn{8}{|c|}{$30^{\circ} \mathrm{C}$} \\
\hline $\begin{array}{l}\text { Incubation } \\
\text { period }\end{array}$ & $\begin{array}{l}3.2 \pm 0.1 \\
93-3.4)\end{array}$ & $\begin{array}{l}3.2 \pm 0.1 \\
(3.1-3.4)\end{array}$ & $\begin{array}{l}2.8 \pm 0.1 \\
(2.7-2.9)\end{array}$ & $\begin{array}{c}2.6 \pm 0.1 \\
(2.4-2.8)\end{array}$ & $\begin{array}{c}2.5 \pm 0.1 \\
(2.3-2.7)\end{array}$ & 0.06 & 0.04 \\
\hline Life cycle & $\begin{array}{c}11.5 \pm 0.1 \\
(11.2-11.7)\end{array}$ & $\begin{array}{c}11.0 \pm 0.5 \\
(10-12)\end{array}$ & $\begin{array}{c}10.0 \pm 0.1 \\
(9.9-10.4)\end{array}$ & $\begin{array}{c}8.8 \pm 0.1 \\
(8.6-8.9)\end{array}$ & $\begin{array}{c}7.8 \pm 0.2 \\
(7.4-8) \\
\end{array}$ & 0.11 & 0.08 \\
\hline Longevity & $\begin{array}{c}16.9 \pm 0.4 \\
(16-17.3)\end{array}$ & $\begin{array}{c}16.0 \pm 0.2 \\
(15.6-16.4)\end{array}$ & $\begin{array}{l}13.5 \pm 0.2 \\
(13-13.8)\end{array}$ & $\begin{array}{c}13.0 \pm 0.3 \\
(12.5-13.5)\end{array}$ & $\begin{array}{c}12.2 \pm 0.4 \\
(11.8-13.0)\end{array}$ & 0.3 & 0.2 \\
\hline Life span & $\begin{array}{c}28.4 \pm 0.3 \\
(27.6-28.8)\end{array}$ & $\begin{array}{c}27.0 \pm 0.6 \\
(26-28)\end{array}$ & $\begin{array}{l}23.5 \pm 0.3 \\
(23-23.9)\end{array}$ & $\begin{array}{c}21.7 \pm 0.3 \\
(21.3-22.2)\end{array}$ & $\begin{array}{c}20.0 \pm 0.4 \\
(19.4-20.6)\end{array}$ & 1.18 & 0.9 \\
\hline
\end{tabular}

B. b. $\mathrm{h}=$ broad bean hay $\mathrm{W} . \mathrm{h} .=$ wheat hay 


\section{Longevity:}

The current study (Table 3 ) indicated that the longevity of the astigmatid mite, $L$. destructor was highly affected in male individuals when the different types of food were used. The statistical analysis of the obtained data showed that L.S. D. at 0.05 level $=0.3$ and 0.2 in case of incubation period for the effect of diets and temperature on this period, respectively. The longevity of this mite averaged the longest period when reared on mixture of broadbean hay with yeast recording 22.0 days at $20{ }^{\circ} \mathrm{C}$, but it sharply decreased to recorded 12.2 days only when mixture of broadbean and wheat hay was introduced as food at $30 \stackrel{\circ}{\circ}$.

\section{Life span:}

During the course of the present investigation, the life span of $L$. destructor male when fed on the previously mentioned diets lasted the shortest period when fed on the mixture of broadbean and wheat hay at $30{ }^{\circ} \mathrm{C}$ and recorded 20 days, as, this period was obviously increased to reached the longest period when the male reared on broad bean hay mixed with yeast used as food at $20{ }^{\circ} \mathrm{C}$. The statistical analysis of obtained results indicated that L.S.D. at 0.05 for effect of diets and temperature was 1.18 and 0.9, respectively, Table (3). Generally, the male individuals took the shortest biological stages (incubation period, life cycle, longevity and life span) than female individuals at different used temperature, and $20{ }^{\circ} \mathrm{C}$ increased these periods. Under controlled conditions about $20 \stackrel{\circ}{\circ}$ and $85 \%$ R.H., Chmielewski (2001) used bruised buckwheat grains as a mite food for $L$. destructor. The life cycle lasted about 17.7 days and the female laid 78.1 eggs per whole lifespan. The results obtained by Cusack et al., 1976; Hallas, 1981; Matsumoto, 1963; Parkinson, 1989; Rack, 1978, Sinha, 1964a,b, 1966; Stratil et al., 1980 give evidence that $L$. destructor accepts not only the grain, seed parts and foodstffs, but also mycelia of fungi infesting them (mouldy products) and might be a vector for saprophytic and pathogenic microorganisms. In this study, from the above mentioned results it can be concluded that the best diets for mass culturing acarid mite, $L$. destructor is a combination of freshly broadbean hay + yeast.

\section{REFERENCES}

Chmielewski, W. (1969). Fauna of mites in stored seeds of sugar beet. Polskie Pismo ent., 39:619-628.

Chmielewski, W. (1977). Powstawanie i znaczenie stadium hypopus $\mathrm{w}$ zyciu $\mathrm{z}$ nadrodziny Acaroida. Prace nauk. Inst. Ochr. Roslin, $28:$ 147-153.

Chmielewski, W. (1987).Parametery rozwuju populacji Glycyphagus destructor (Schr.) (Acarida, Glycyphagidae) - gatunku roztoczy spotykanego w ulach pszczelich. Pszczelich. Zesz, Nauk. 31: 213-221 (in Polish)

Chmielewski, W. (2001). Buckwheat as a nourishment of Lepidoglyphus destructor (Schr.) (Acari: Glycyphagidae). Fagopyrum, 18:61-64.

Cornete, C. and O. Kahl (1996). Decreased developmental reproductive potential in Lepidoglyphus destructor after a prolonged hypopus stage. Third Symposium of the European Association of Acarologists (EURAAC), 1-5 July, 1996, Amsterdam. The Northlands (Abstracts):78.

Cusack, P.D., G.O. Evans and P.A. Brennan (1976). The origin and sources of mite infestation of stored grain and related products in the Republic of Ireland. Ann. Appl. Biol., 82:178-179.

Duncan, D. B. (1955). Multiple range and multiple F.test. Biometrics, 11: 1-42.

Griffith, D.A. 1960.Some field habitats of mites of stored food products. Ann. Appl. Biol., 48:133-134.

Hallas, T.E. (1981). Mites of stored hay in Ireland. J. Agr. Res. Icel.13:61-67.

Harein, P. and R. Meronuck (1995). Stored grain losses due to insects and molds 
and the importance of proper grain management, In V. Krischik, G. Cuperus \& D. Galliart, pp 29-31. E-912.CES. Div. Agric. Sci. Nat. Res.OSU.USDA. FGIS. ES. APHIS.242 pp.

Hubert, J., V. Stejskal, Z. Munzbergova, A. Kubatova, M. Vanova and E. Zdarkova (2004). Mites and fungi in heavely infested stores in Czech Republic. J. Econ. Entomol, 97: 2144-2153.

Hughes, A. M. (1976). The mites of stored food and houses. Tech. Bull., Min. Agric. and Fisheries in London, 63: 145 pp.

Knulle, W. (1991). Genetic and environmental determinants of hypopus duration in the stored-product mite Lepidoglyphus destructor. Exp. \& Appl. Acrol., 10:231-258.

Kondreddi, P.K., B.L. Elder, B.L. VyszenskiMoher and L.G. Arlian (2006). Importance of sensitization to Tyrophagus putrescentiae in the United States. Ann. Allergy Asthma Immunol. 96 (1): $124 \mathrm{pp}$.

Matsumoto, K. (1963). Studies on the environmental factors for the breeding of grain mites Part IV Comparison of the effects of humidity and temperature on the breading of the grain mites, Tyrophagus dimidiatus, Aleuroglyphus ovatus and Glycyphagus domesticus. Jap. J. Sanit. Zool., 14: 82-88.

Parkinson, C. L. (1989). Population increase and damage by three species of mites on wheat at $20{ }^{\circ} \mathrm{C}$ and two humilities. Exp. \& Appl. Acarol., 8:179-193.

Rack, G. 1978. Milben in Vorraten, ihre Einfubrung fur das Studium und fur den praktischen Gebrauch. Min. Agric. \& Plant Protection Organization. Appendix Bull. No.26, 42 pp.

Sanchez-Ramos, I. and P. Castanera (2003). Laboratory evaluation of selective pesticides against the storage mite Tyrophagus putrescentiae (Acari: Acaridae). J. Med.Entomol., 40: 475481.

Sheals, J.G. (1956). Notes on a collection of soil Acari. Ent. Mon., Mag. 92,:99-103.

Sinha, R.N. 1964a. Mites of stored grin in Western Canada, ecology and survey. Proc. Entomol., Soc. Man. 20: 19-33.

Sinha, R.N. (1964b). Ecological relationships of stored-products mites and seed-born fungi. Acarologia, 6:372-389.

Sinha, R.N. (1966). Feeding and reproduction of some stored-product mites on seed-borne fungi. J.Econ. Entomol., 59: 1227-1232.

Stratil, H. U., H.H. Strattil and W. Knule (1980). Untersuchungen uber die spezifische Vermehngsrate von Populationen der im Lagergetreide lebenden Milbe Glycyphagus destructor (Schrank, 1781) bei verschiedenen Temperatur und Luftfeuchtebedingungen.Z. Ang. Ent., 90: 209-220. 
Biological aspects of lepidoglyphus destructor (schrank) (acari: astigmata:....

(Schrank) (Acari: Lepidoglyphus destructor المظاهر البيولوجية للاكاروس Astigmata: Glycyphagidae)

عصام محمد عبدالسلام ياسين ، اسماء رضا عبدالخالق ، ممدوح محمد السباعى ، صافيناز احمد عبدالعزيز

معهد بحوث وقاية النباتات - مركز البحوث الزراعية - الدقى - جيزة - مصر

الملخص العربى

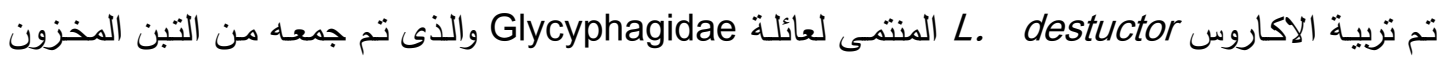

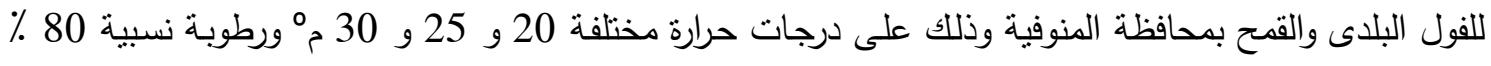
عند التغذية على تبن الفول البلدى وتبن القهـح بصورة منفردة وفى صورة خليط معا او مـع الخميرة الجافة حيث

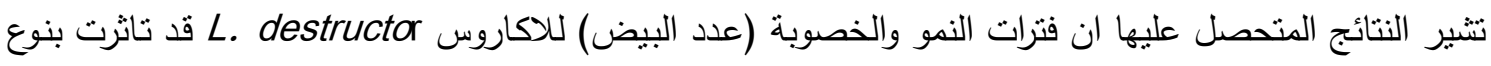

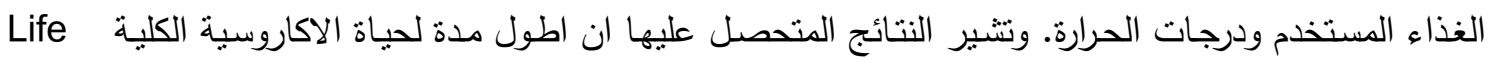
قد سجلت زمنا مقداره 49.4 يوما عندما تغذت الافراد الاناث على مخلوط تبن الفول البلدى مع الخميرة عند

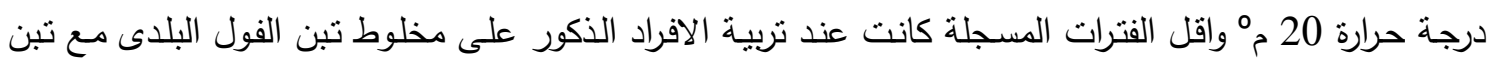

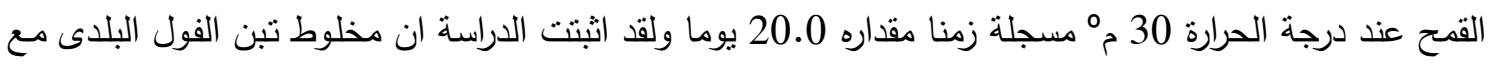
الخميرة الجافة كانت افضل التغذية المستخدمة لوضع البيض عند 30 مْ حيث وضعت الانثى عليه عددا مقداره 159.9 بيضة واقل الاغذية كانت مخلوط تبن الفول البلدى مع تبن القمح ووضعت الانثى عليه 82.2 بيضة فقط عاند عند التربية على 20 مْْ. وبوجه عام اتضح ان الافراد الذكور تستغرق زمنا اقل من الاناث لهذا الاكاروس اثناء

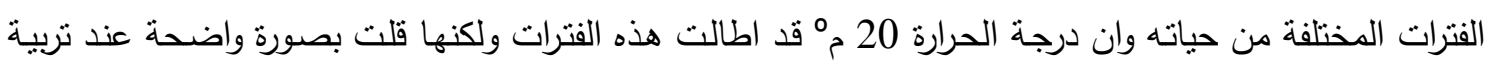
الاكاروس عند درجة الحرارة 30 مْْ. 